\title{
SCHLAFEN, ESSEN UND REDEN: SYMBOLISCHE BINDUNG
}

\section{ORIGINALER ARTIKEL}

PALLADINO, Ruth Ramalho Ruivo1, SOUZA, Luiz Augusto de Paula², PALLOTTA, Mara Lucia ${ }^{3}$, COSTA, Rogério da ${ }^{4}$, CUNHA, Maria Claudia ${ }^{5}$

PALLADINO, Ruth Ramalho Ruivo. Et al. Schlafen, Essen und Reden: symbolische Bindung. Revista Científica Multidisciplinar Núcleo do Conhecimento. Jahrgang 06, Ed. 08, Vol. 06, S. 153-170. August 2021. ISSN: 2448-0959, Zugangslink: https://www.nucleodoconhecimento.com.br/psychologie/symbolischebindung, DOI: 10.32749/nucleodoconhecimento.com.br/psychologie/symbolischebindung

\section{ZUSAMMENFASSUNG}

Schlaf, Essen und Sprache sind Säulen des gesunden Lebens von Kindern, von Geburt an miteinander verflochten und bilden die dynamische Struktur der kindlichen Entwicklung. Dies sind die Auswirkungen von voneinander abhängigen Bedingungen: organisch, psychisch und sozial, die das Kind einbeziehen und gleichzeitig aus organischen und symbolischen Erbschaften resultieren. Letzteres überbestimmt und moduliert die Interaktion des Kindes mit der Umwelt, insbesondere mit dem anderen Menschen, der dort ist. Dieses Erbe zeichnet Verhaltens- und

\footnotetext{
${ }^{1} \mathrm{PhD}$ in Klinischer Psychologie, PhD Professor des Postgraduiertenprogramm in Menschliche Kommunikation und Gesundheit an der Fakultät für Humanwissenschaften und Gesundheit der PUC-SP (ORCID: https://orcid.org/0000-0001-8466-838X).

${ }^{2} \mathrm{PhD}$ in Klinischer Psychologie, ordentlicher Professor des Postgraduiertenprogramm in Menschliche Kommunikation und Gesundheit an der Fakultät für Humanwissenschaften und Gesundheit der PUC-SP (ORCID: https://orcid.org/0000-0003-4968-9753).

${ }^{3}$ Doktorand in Menschliche Kommunikation und Gesundheit bei PUC-SP (ORCID: https://orcid.org/0000-00015986-9657).

${ }^{4} \mathrm{PhD}$ in Philosophie, PhD-Professorin des Graduiertenprogramms in Kommunikation und Semiotik an der PUCSP (ORCID: https://orcid.org/0000-0002-6807-4263).

${ }^{5} \mathrm{PhD}$ in Klinischer Psychologie, ordentlicher Professor des Postgraduiertenprogramm in Menschliche Kommunikation und Gesundheit an der Fakultät für Humanwissenschaften und Gesundheit der PUC-SP (ORCID: https://orcid.org/0000-0003-3198-6995).
}

RC: 98237

Verfügbar in: https://www.nucleodoconhecimento.com.br/psychologie/symbolischebindung 
Verhaltensmuster, die oft zu Veränderungen beitragen können, die bis zu einem gewissen Grad die Gesamtentwicklung des Kindes beeinträchtigen. In der Kinderklinik umfasst die Beschreibung der Entwicklungsstörungen, von den mildesten bis zu den schwersten, in der Regel Ernährungs-, Schlaf- und Sprachaspekte, was dann eine Basistriade nahelegt, die die Kliniker nach der Möglichkeit befragt, dass es mehr als einen einfachen Zufall gibt, eine Korrelation zwischen grundlegenden biologischen Funktionen. Wenn dies der Fall ist, wird es für den Kliniker wichtig sein, sich diese Perspektive anzueignen, da die Implikation wahrscheinlich Besonderheiten in den Diagnose- und Behandlungsverfahren bestimmen wird. In dieser Richtung lohnt es sich, die Entwicklung dieser Funktionen (Schlaf, Ernährung, Sprache) zu vertiefen und zu diskutieren, um ihre konstitutive Korrelation, die Verbindung zwischen ihnen, zu klären.

Schlüsselwörter: Sprache, Essen, Schlaf.

\section{EINLEITUNG}

Schlaf, Essen und Sprache sind Säulen des gesunden Lebens von Kindern, von Geburt an miteinander verflochten und bilden die dynamische Struktur der kindlichen Entwicklung.

Eine solche Verflechtung ist jedoch kein einstimmiges Postulat, in den Projekten zur Beschreibung und zum Verständnis dieser Triade oder jeder Teil wird separat genommen oder dann wird auf das Privileg einer Partei gegenüber der anderen hingewiesen, das heißt, es wären Beziehungen, die nicht als Implikation definiert werden können.

Um über die Implikation nachzudenken, ist es notwendig anzunehmen, dass Schlafen, Essen und Sprechen den Körper betreffen, aber einen Körper, der einen Namen verlangt, einen subjektiven Körper und daher einen Körper, der durch das Symbolische angeworben wird: In der Verbindung des Eigennamens und des

Verfügbar in: https://www.nucleodoconhecimento.com.br/psychologie/symbolischebindung 
Körpers gibt es bei der Lektüre dieses Merkmals etwas, das sich durch die Aneignung von sich selbst artikuliert, was kein so offensichtliches und einfaches Element in der menschlichen Konstitution ist (LEITE, 2008, S.16). Wie wird eine solche Aneignung des Körpers dargestellt, der aus seinem eigenen Körper kommt, wenn er ernannt wird? Den Körper zu benennen heißt, ihn im symbolischen Feld zu erkennen, Wirkung unendlicher Artikulationen, Handlungen und verflechten.

Schlaf-, Ernährungs- und Sprachmuster sind Auswirkungen von voneinander abhängigen Zuständen: organisch, psychisch und sozial, die das Kind einbeziehen und gleichzeitig aus organischen und symbolischen Erbschaften resultieren. Letzteres überbestimmt und moduliert die Interaktion des Kindes mit der Umwelt, insbesondere mit dem anderen Menschen, der dort ist.

Für Babys kann diese Umgebung privilegiert durch die mütterliche Figur, die Mutter und, es ist erwähnenswert, nicht einmal unbedingt die biologische Mutter oder Betreuer dargestellt werden, sondern eine asane mütterliche Instanz, ein anderer Mensch, der die "nächster Helfer", den Freudschen Nebensmench, einschreibt, wie Cabassu (2003) erklärt. Derjenige, der nicht nur das Überleben des kindlichen Organismus garantiert, sondern inn als Subjekt anerkennt, bildet die Verbindung zwischen Verlangen (mütterlich) und Wort, von der subjektivität und soziale Beziehungen möglich werden und für das Baby beginnen, indem er seinen Körper und seine Inschrift in einem Text der Zugehörigkeit und Geselligkeit benennt.

Wenn, wie gesagt, Schlaf, Nahrung und Sprache Säulen des kindlichen Lebens sind, hängt ihre Bedeutung daher von mütterlichen Äußerungen ab, die an es gerichtet sind, aber nur, wenn sie - den Hunger stillen, sich von der Kälte erwärmen, in den Schlaf schaukeln... - werden von einem nicht-anonymen Wunsch begleitet (STORK, LY, MOTA, 1997, S. 34): Hier ist die Gegenwart des Anderen, die Institution der mütterlichen Beziehung.

$\mathrm{RC}: 98237$

Verfügbar in: https://www.nucleodoconhecimento.com.br/psychologie/symbolischebindung 
Darüber hinaus ist es wichtig zu wissen, dass es notwendig ist, in der mütterlichen Haltung eine ebenso entscheidende kulturelle Bedeutung anzuerkennen, denn Mütter interpretieren und reagieren auf die [...] Manifestationen des Babys gemäß den Normen der Gesellschaft, zu der sie gehören, sogar wenn ihre Reaktionen auch durch die persönliche psychische Dynamik moduliert werden (CISMARESCO, 1997, S. 267).

Dieser Zustand setzt im weiteren Sinne die Zentralität der Funktion einer Kontinentfamilie voraus, für die der Begriff "Ich-Haut" von Anzieu (1989) aufschlussreich sein kann, da er sich auf die Grenzen zwischen dem Biologischen und dem Symbolischen bezieht und für das Baby seine Etablierung auf das Bedürfnis nach einer naristischen Hülle reagiert und dem psychischen Apparat die Gewissheit und Beständigkeit eines grundlegenden Wohlbefindens sichert (op. cit., p. 44). Dieses Gefühl der Sicherheit wird grundlegend für das Gefühl der Zugehörigkeit sein, grundlegend für die Konstruktion der Identität des Kindes.

Dieses gleichzeitig organische und symbolische Erbe, das in den Beziehungen des Kindes zum anderen übertragen wird, das natürlich mit organischem Erbe verbunden ist, wird Verhaltensmuster und Verhaltensweisen zeichnen, die oft zu Veränderungen beitragen können, die bis zu einem gewissen Grad die allgemeine Entwicklung des Kindes beeinträchtigen.

In der Kinderklinik umfasst die Beschreibung der Entwicklungsstörungen, von den mildesten bis zu den schwersten, in der Regel Ernährungs- und Schlafaspekte (WINNICOTT, 1975; MADEIRA, AQUINO, 2003 ; SANTOS, 2004; JERUSALINSKY, 2004).

Die Tatsache, dass diese Symptome fast immer aufeinander abgestimmt sind, stellt Kliniker in Frage, ob es mehr als nur einen einfachen Zufall zu einer Korrelation zwischen grundlegenden biologischen Funktionen gibt.

RC: 98237

Verfügbar in: https://www.nucleodoconhecimento.com.br/psychologie/symbolischebindung 
Darüber hinaus sind in den klinischen Beschreibungen von Sprachentwicklungsstörungen Berichte über Fütterungsprobleme (PALLADINO, CUNHA, SOUZA, 2007) und Schlaf häufig, was auf einen Dreiklang von Korrelationen hindeutet.

Die verschiedenen Entwicklungsbereiche teilen jedoch oft nicht die Idee einer signifikanten Korrelation zwischen solchen Funktionen, und es ist üblich, dass Schlaf-, Fütterungs- und Sprachprobleme getrennt betrachtet werden. Wenn dies der Fall ist, wird bei Vorliegen von Entwicklungsveränderungen das Phänomen als Komorbidität angenommen.

Es gibt Ausnahmen und eine solche Vorherrschaft, es ist eine Tatsache, mit Relevanz für die Psychoanalyse und bestimmte Ansätze der Psychologie (GROMANN, 2002), sowie für den kleinen Teil der Studien von Disziplinen, die mit beiden im Dialog stehen, wie Sprachtherapie, psychiatrische Medizin, Neurologie und Endokrinologie. Diese Ausnahmen regten zu weiteren Überlegungen an.

Die Annahme der Funktionszusammenhänge ermöglicht es, die symptomatische Ausrichtung zwischen Ess-, Schlaf- und Sprachsymptomen in der frühen Kindheit zu klären. Wenn dies der Fall ist, wird es für den Kliniker wichtig sein, sich diese Perspektive anzueignen, da die Implikation wahrscheinlich Besonderheiten in den Diagnose- und Behandlungsverfahren bestimmen wird.

In dieser Richtung lohnt es sich, die Entwicklung dieser Funktionen (Schlaf, Ernährung, Sprache) zu vertiefen und zu diskutieren, um ihre konstitutive Korrelation, die Verbindung zwischen ihnen, zu klären.

$\mathrm{RC}: 98237$

Verfügbar in: https://www.nucleodoconhecimento.com.br/psychologie/symbolischebindung 


\section{ESSEN UND SPRACHE: DIE ÜBERRASCHUNG IN DER BEOBACHTUNG}

Vor einiger Zeit, im täglichen Leben der Kinderklinik, begann eine Beobachtung der Indizienkraft uns in Frage zu stellen.

Dies geschah angesichts wiederholter elterlicher Erzählungen über Essprobleme in den in Evaluationsverfahren genannten Fällen oder sogar bei der Nachverfolgung von Therapieplänen bei Kindern mit verschiedenen Sprachentwicklungsproblemen. Im Umgang mit diesen Kindern kam es früher oder später zu Beschwerden über die Fütterung, die von Eigenheiten zu Schluckstörungen übergingen, und ihre beharrliche Wiederholung gewann ihren Platz in unserem klinischen Hören und damit gewann der Indizienwert der Beobachtung an Relevanz.

Basierend auf diesen Beobachtungen strukturierten wir die Forschung mit einer Stichprobe von 35 Patienten, und die erhaltenen Beweise klärten wichtige Begleiterscheinungen zwischen Sprach- und Essproblemen, was auf eine signifikante Korrelation zwischen innen hindeutet (PALLADINO, CUNHA, SOUZA, 2004 und 2007).

Eine psychoanalytische Lektüre des Themas, die von der Revista Francesa de Ortofonia (2004) angeboten wurde, ermöglichte die theoretische Abdeckung der Ergebnisse dieser Forschung, was uns veranlasste, über den Zusammenhang zwischen Sprache und Essproblemen unter dem Begriff der Oralität nachzudenken, wie er von vorgeschlagen wurde Thibaut (2006, S. 115): die orale Zone ist eine der körpererogenen Zonen, dh ein vom Trieb getragener Raum, in dem viele Funktionen in die gemeinsame Ebene symbolischer Funktionen gemischt werden. Der Mund (Organ) ist in diesem Sinne das Territorium der Nahrung, der Sprache und der Zuneigung.

RC: 98237

Verfügbar in: https://www.nucleodoconhecimento.com.br/psychologie/symbolischebindung 
Mit anderen Worten, die mündliche Zone ist das somatische Feld, in dem Oralität als psychische Ebene symbolisch Nahrung und Sprache miteinander verwebt.

\section{ESSEN, SPRACHE UND SCHLAF: EINE NEUE ARTIKULATION}

Diese konzeptionelle Neupositionierung lenkte und entfaltete unser klinisches Hören auf andere Räume und führte uns in jüngerer Zeit dazu, die Beziehung von Essen und Sprache aus der Einbeziehung des Schlafes zu überarbeiten und eine konstitutive Triade des Kindes zu bilden. Der Schlaf wurde auch einbezogen, weil er auch der Protagonist in der grundlegenden Szene der Konstitution des Kindes ist (PALLADINO, 2016, 2018). Schlaf ist mit der Babyfütterungsszene verbunden und ist Teil der elterlichen Erzählungen bei Kindern mit Sprachentwicklungsproblemen, obwohl sie immer noch undurchsichtig sind, dh als wertlos oder mit geringem Indizienwert in Bezug auf das Risiko für die kindliche Entwicklung angesehen werden.

Unter der Annahme der konstitutiven Korrelation zwischen diesen Funktionen wird es notwendig sein, die beobachtete symptomatische Ausrichtung zwischen Ess-, Schlafund Sprachsymptomen zu klären sowie mögliche Implikationen für das Diagnoseund Behandlungsverhalten in diesen Fällen zu analysieren.

Der Schlaf, eine grundlegende biologische Funktion, die für die Wiederherstellung des Energiestoffwechsels des Gehirns und die Gedächtniskonsolidierung (CABALLO, NAVARRO, SIERRA, 2002) sowie für das psychische Gleichgewicht selbst wichtig ist, resultiert aus einer allmählichen zeitlichen, strukturellen und physiologischen Organisation des Schlafes -Wach-Rhythmus (GEIB, 2007; PIAULINO DE ARAÚJO, 2012.) Es ist ein Zustand der Gehirnfunktion mit zwei verschiedenen und messbaren Phasen: REM-Schlaf (Rapid Eye Movement) und NREM-Schlaf (No Rapid Eye Movement). Die Unterschiede liegen hauptsächlich im Hinblick auf metabolische Mechanismen mit daraus resultierenden Veränderungen in physiologischen Prozessen und Haltungsbedingungen. Im REM-Schlaf kommt es zu

$\mathrm{RC}: 98237$

Verfügbar in: https://www.nucleodoconhecimento.com.br/psychologie/symbolischebindung 
einer Erhöhung des Stoffwechselniveaus, einer bestimmten Muskelatonie, einer Verringerung der Körpertemperatur, einem ausgeglichenen Atemrhythmus (mit wenigen und kurzen Apnoen), schnellen Augenbewegungen und bei Kindern kann es zu Weinen/Lächeln/Stöhnen kommen . Im NREM-Schlaf kommt es zu einer Abnahme des Stoffwechselniveaus, einer Abnahme der Atemfrequenz und der Körpermobilität (JOHN, 2000). In dieser Phase gibt es eine umfangreiche Arbeit der Zellregeneration und in der anderen, der REM-Phase, hauptsächlich psychologische Regeneration, da hier die meisten Träume stattfinden.

REM- und NREM-Schlaf wechseln sich ab, ebenso wie Schlaf- und Wachzustände. Der Zyklus zwischen Schlaf- und Wachzuständen, der zu Beginn nur biologisch bestimmt ist, beginnt noch in der fetalen Phase organisiert zu werden und seine Konstitution ist eng mit der Entwicklung des Zentralnervensystems verbunden (GEIB, 2007). Allmählich leidet diese Organisation unter den Auswirkungen exogener Reize wie intensiver Leuchtkraft, unterschiedlicher Geräusche und vor allem der menschlichen Präsenz, wodurch der Schlaf-Wach-Zyklus einen Rhythmus hat, der durch diese doppelte Interferenz gekennzeichnet ist - endogen und exogen.

Bei der Geburt ist das Schlafmuster des Babys - im Wesentlichen physiologisch einfacher, mit zwei Zuständen, aktiv (REM-Schlaf) und ruhig (NREM-Schlaf), in einem Schlaf-Wach-Rhythmus, der als ultradiane bezeichnet wird und noch nicht von Tag-Nacht-Wechsel dominiert wird. Die Zyklen wechseln sich in kürzerer Zeit ab, und in den ersten Monaten ist der aktive Zustand vorherrschend: Das Baby schläft jederzeit und wacht sehr leicht auf, und die Wachheit wird durch Weinen angekündigt und erst später wird die stille Wachheit beobachtet. Im Laufe der Zeit wird dieser Rhythmus von der Umgebung modifiziert, was zu einer anarchischen Atytypisierung führt, dh zu Beginn völlig unorganisierten veränderungen und des desorganisierten Schlafzyklus, nicht nur durch die leuchtenden und hörbaren Reize, sondern hauptsächlich durch menschliches Handeln, das am Ende den Rhythmus moduliert,

$\mathrm{RC}: 98237$

Verfügbar in: https://www.nucleodoconhecimento.com.br/psychologie/symbolischebindung 
der schließlich zu Cycardiano wird, mit langen Nachtperioden des ruhigen Schlafes oder noch ruhigerer Wachheit. (PIAULINO DE ARAÚJO 2012; JOHN, 2000)

Eine vorherrschende menschliche Handlung in der Frage des Schlafes von Babys ist die Fütterung, aus zwei Hauptgründen: Es geht oft dem Moment voraus, in dem das Baby schläft, neben der Tatsache, dass es sich auf einen anderen Menschen für seine Wirksamkeit verlässt. So beginnt das, was zu Beginn eine Bestimmung nur endogen hat, unter dem Einfluss der äußeren Umgebung zu leiden. Hier konsolidieren sich die ultradianischen und cycardianischen Rhythmen und beginnen sich abzuwechseln, wobei der erste die Verteilung der Schlafphasen und der zweite die Zustände von Schlaf und Wachheit steuert, Schlaf in der Nacht und Wachheit am Tag gewinnt (PIAULINO DE ARAÚJO, 2012; JOHN, 2000).

Indem sie das Baby auf den Schoß nimmt, um es zu stillen, bietet inm die Mutter ein unvergleichliches affektives Feld: das Vergnügen, die Milch zu sispeln, die Worte zu hören, das Reiben des Körpers des anderen zu erleben, diese Freude lässt inn einschlafen, um sich selbst zu geben.

Schlaf und Nahrung liegen somit jenseits der Aufzeichnung der Notwendigkeit und stellen darüber hinaus eine endgültige Verflechtung dar (PENHA, 2002; FÉDIDA, 1977). Wie bereits erwähnt, hängen diese Funktionen des Babys für ihre gebührende Verwirklichung von einem anderen Menschen ab und erhalten aus diesem Grund symbolischen Charakter, der sich als psychische Erfahrungen anpasst. Solche Erfahrungen, im Allgemeinen des Vergnügens, hinterlassen psychische Spuren und Gehirntritte und schaffen kraftvolle Erinnerungen, die die Probanden zur Geselligkeit mit dem anderen einladen.

Die Mutter stillt ihr Baby, spricht und / oder singt mit inm, streichelt es, was zusammen mit dem Sättigungsgehalt das Kind beruhigt und den Schlaf fördert. Der Hunger wird jedoch einige Zeit später das Baby wecken und den Wach-Schlaf-Zyklus wiederholen.

RC: 98237

Verfügbar in: https://www.nucleodoconhecimento.com.br/psychologie/symbolischebindung 
Es bedeutet, dass dieser kontinuierliche Zyklus nicht nur eine Wirkung des organischen Rhythmus sein wird, sondern auch eines psychischen Rhythmus, der von der Mutter gedruckt wird (FARIAS, 2004), durch ihre Fürsorge.

Mit anderen Worten, die Brust (oder die Flasche) wird zum Austauschfeld und zum Schlafplatz (FOLINO, LOPES DE SOUZA, 2013), Sprechen/Summen und Schaukeln bilden das ethos dieser Schlafpraktiken. Laznik-Penot (1997, S. 37) zeigte die entscheidende Bedeutung von mütterlichen Melodien - der Musik der Mutterstimme, die mit dem Baby spricht. Diese nährende Szene wiederum impliziert Nahrung, gibt ihr auch eine symbolische Existenz.

Letztendlich wird dieses intime und wiederholte Ritual, das Körper und Worte, Schlaf und Essen beinhaltet, die grundlegende Szene der Humanisierung bilden und den Beziehungsplan bilden, in dem die Bindung zwischen dem Baby und seiner Mutter eingeweiht wird. Tatsächlich bestätigt diese Perspektive Spinozas Ideen (2007) über Zuneigungen und ihre Folgen in Bezug auf Freude oder Traurigkeit: Mutter und Baby beeinflussen und werden voneinander beeinflusst, was ein Netzwerk von affektiven Verbindungen bildet, die sie als solche konstituieren und oft die Macht erweitern, in dieser einzigartigen Beziehung und in allen anderen zu handeln. Die erhöhte Handlungskraft in der Welt ist das, was Spinoza Freude nennt.

Die Zuneigung zwischen Mutter und Baby ist jedoch nicht immer potenzgemärt, manchmal kann das Gegenteil passieren. Schauen wir uns ein relativ häufiges Beispiel an: Mütter, die Baby-Blues durchmachen (gutartige Depression, vorübergehend, potenziell produktiv, weil es Teil der Veränderungen ist, die durch das Kommen eines Babys erzeugt werden) und nicht die Möglichkeit der Ausarbeitung und Akzeptanz durch den sozialen Kontext finden, der die Geburt eines Kindes oft nur durch die Gefühle von Glück und Fülle erkennt, die kulturell, es sollte produzieren (FOLINO, LOPES DE SOUZA, 2013).

$\mathrm{RC}: 98237$

Verfügbar in: https://www.nucleodoconhecimento.com.br/psychologie/symbolischebindung 
Diese depressiven Mütter verbringen weniger Zeit damit, ihre Babys zu suchen, zu berühren, mit ihren Babys zu sprechen, weniger Reaktionsfähigkeit, Spontaneität und niedrigere Aktivitätsraten mit ihren Babys zu zeigen. Postpartale Depression trägt dazu bei, dass die Dyade asynchron wird, so dass die Mutter wenig oder nicht ansprechbar ist (SERVILHA, RAAD BUSSAB, 2015). Während dieser Zeit erhöht der affektive Prozess zwischen Mutter und Baby nicht die Potenz beider, im Gegenteil, er reduziert sie und kann in einigen Fällen sogar psychische Probleme oder Störungen für Mütter und für die Entwicklung des Babys entfalten. Die Abnahme der Handlungskraft ist wiederum das, was Spinoza Traurigkeit nennt.

Es ist von grundlegender Bedeutung, dass der Fütterungs-Acarcer-Schlaf-Zyklus für das Baby vielfältige psychische Erfahrungen darstellt, die für das Baby mehrdeutige Sinne implizieren: Fütterung und Akarzeration werden zu Raum und Zeit der MutterBaby-Interaktion und der Schlaf der Trennung zwischen innen - beim Einschlafen wird das Baby in die Krippe gelegt. Schlafen stellt einen Schnitt, ein Intervall oder eine Diskontinuität in der ursprünglichen Verbindung zwischen dem Kind und seiner Mutter dar. Das Ritual enthält an sich die Mehrdeutigkeit zwischen Akzeptanz und Trennung. Diese Position der Mutter, doppelt, mehrdeutig, zart, wird inszeniert, wenn sie singt und das Kind einschläft, um in die Wiege gelegt zu werden: liebevolle Annäherung und Trennung.

Nach und nach renspäht die Mutter die Schläge - eine Gelegenheit, den Wachzustand zu festigen - und führt Ersatzstoffe ein, die zwischen ihrem eigenen Körper und dem des Babys als Ersatz für ihre Anwesenheit stehen: Schnuller, Pfannen und Spielzeug, sogar das Wort metaphorisch, das die Mutter bringt, repräsentiert sie zum Baby. Diese Ersatzstoffe werden eingefügt, um Verschiebungen in der Fusionsbeziehung zu wirken, die die frühen Tage der MutterBaby-Bindung ausmacht, sind Unterstützungen für das Baby, um sich der Angst der Trennung zu stellen, die der Schlaf ankündigt, das Gefühl, dass Schlaf die

Verfügbar in: https://www.nucleodoconhecimento.com.br/psychologie/symbolischebindung 
Abwesenheit der Mutter bestimmt, Entzug der Umgebung, die ihr Leben und Vergnügen sichert.

Es sollte beachtet werden, dass die Notwendigkeit, pendeln zum Einschlafen zu betreiben, im Erwachsenenalter beibehalten und aktualisiert wird, in den Ritualen, die der Schlafenszeit vorausgehen: Bäder, Tees, Lesungen .... Es ist, als ob diese Rituale die "Gefahr" beseitigen, die die Einsamkeit des Schlafes mit sich bringt, und eine Art Versicherungsschutz für Taubheit garantieren. Mehrere ältere Menschen haben Schwierigkeiten beim Einschlafen aus Angst vor dem Tod (GEIB, et al, 2003): Angst vor dem Verlust der eigenen und der Welt. Der Erwachsene hat die verinnerlichte Funktion der mütterlichen Asseguradora-Instanz, die Die Verschiebungen bedient, die Ersatzstoffe desjenigen geschaffen, der der erste Wächter des Schlafes war, jetzt unbewusst wiederbelebt, zum Beispiel mit Massagen, sinnlichen Praktiken, Einnahme von Essen, Getränken oder Drogen.

Während der Kindheit, in jeder Kultur und aufgrund der sozialen und wirtschaftlichen Bedingungen unterschiedlich, gibt es mehrere Möglichkeiten, diese Schlafschmerz (sowohl von Mutter als auch von Baby) zu umgehen: Ein Doppelbett (ganz oder teilweise), ein Mehrbettzimmer und das Vorhandensein von (notwendigem) Nachtstillen (BLAIR, 2008).

Aber es ist wichtig zu beachten, dass Kliniker und Forscher dazu neigen, sich auf den sogenannten "Goldstandard" des Säuglingsschlafs in den westlichen Gesellschaften zu beziehen, der so genannt wird, ein Muster, das früh gesucht wird: Einzelbett, getrenntes Zimmer von den Eltern, Fehlen von nächtlicher Ernährung.

Es gibt jedoch verschiedene Warnungen in Bezug auf das Thema Schlaf, da es auch die Teilnehmer der Szene und Schlafgewohnheiten importiert, konstitutiv außerhalb von Schlafzyklen (GEIB, 2007). Minimal wird angenommen, dass es für ein Kind, das nicht einschläft oder aufeinanderfolgende Erweckungen hat, eine Mutter geben kann,

$\mathrm{RC}: 98237$

Verfügbar in: https://www.nucleodoconhecimento.com.br/psychologie/symbolischebindung 
die nicht daran denkt, ihren Körper vom Körper ihres Babys zu trennen, aufzuhören, mit ihm zu sprechen, für ihn zu summen, ihn zu schnitzen...

Eine Möglichkeit, diese Angst zu überwinden, ist das Verlangen nach dem Traum, ein Fall, in dem das, was verloren ging oder fehlte, oniral aufsteigen kann.

Anfangs postulierten die Studien, dass Träume eine exklusive Aktivität der REMPhase des Schlafes darstellten. Neuere Forschungen zeigen jedoch, dass es keine Exklusivität gibt, obwohl diese Aktivität in der REM-Phase vorherrscht, wobei Wissenschaftler erkennen, dass sie sicherstellen soll, dass der Schlaf seine regenerierende Funktion erfüllt (GROMANN, 2002).

Träume sind eine Wirkung der Taubheit der Zensur, die Empfindungen, Gefühle und Handlungen verhindern; Zensur, die den Verstand aller bewohnt und sowohl die Wachheit als auch den Schlaf selbst stört. Die Taubheit schafft Raum für das, was man Traummachen nennt, das heißt, die Konstruktion von Gedanken frei von Verboten. Aus diesem Grund ist es so, dass Träume psychische Wiederherstellung durchführen, so wichtig wie physische Wiederherstellung, die hauptsächlich in der NREM-Phase betrieben wird.

In der Entwicklung des Kindes werden die Schlafphasen allmählich befolgt, um den Schlaf richtig zu organisieren, was inn zu einer Leinwand für Träume macht: vom physiologischen zum symbolischen Schlaf. Das Baby muss den Weg zwischen Essen, Schließen der Augen, Verlieren seiner Mutter (außer Sichtweite!) und treffen Sie sie wieder in Träumen (PENHA, 2002).

Was den Schlaf betrifft, gibt es einen Goldstandard für Lebensmittel, die hauptsächlich kulturell produziert werden (auch wenn dort organische Aspekte berücksichtigt werden). Im Westen: Stillen für mindestens sechs Monate, natürliche Entwöherung, Einführung von Pasten und Feststoffen, ein Weg, der schnell von

RC: 98237

Verfügbar in: https://www.nucleodoconhecimento.com.br/psychologie/symbolischebindung 
warmer Flüssigkeit mit unbestimmtem Geschmack zu festem, heißem, salzigem Essen in vertrauten Szenen mit individueller Leistung gehen muss.

Wie beim Thema Schlaf stehen jedoch mehrere Inhalte auf dem Spiel, die die Szene individualisieren und Aufmerksamkeit verdienen (JERUSALINSKY, 2004; MADEIRA, AQUINO, 2003).

Es ist möglich, eine Parallele zu ziehen und zu sagen, dass für ein Kind, das weint, würgt, Rumina, Erbrochenes und / oder Essen ablehnt, zusätzlich zu möglichen anatomophysiologischen Problemen, es eine Mutter geben kann, die Schwierigkeiten hat, sich als die andere Versichererin des Subjekts in der Konstitution zu positionieren, die Essensszene unsachgemäß ausführt, ohne Freude oder sogar mit Traurigkeit.

Eine metaphorische Szene dieser Schwierigkeit wird in der Entsöherung dargestellt, einem allmählichen Prozess, der Feinkost verdient, da er durch symbolische Trennungsoperationen aufrechterhalten wird und daher kein "natürliches" Ereignis ist, offensichtlich, dessen Versorgung mit Nahrung und der Übergang bestimmter Arten zu anderen immer ruhig sind.

\section{DIE STREICHUNG DER SPRACHE}

Sprache ist die menschliche Handlung, die bei Schlaf- und Fütterungsproblemen vorherrscht. Beide Funktionen stellen symbolische Erfahrungen in den Worten der Mutter und in den kindlichen Worten dar, die sich die Mutter vorstellt. Im Lied, in der Geschichte, in den Einwürfen der Mutter und im Zucker, im Blick, im Augenschluss, in den unartikulierten Klängen, die die "Äußerungen" des Babys sind. Die dialogische Szene ist als "Wortspiel" in baktinischer Interpretation gemacht. Ein Gründungsdialog, der fortbesteht und den Eintritt des Subjekts in die Sprache markiert, ein Territorium der Begegnungen und Konfrontationen zwischen Subjektivitäten: polyphones Aufeinanderprallen verschiedener sozialer Instanzen,

$\mathrm{RC}: 98237$

Verfügbar in: https://www.nucleodoconhecimento.com.br/psychologie/symbolischebindung 
widersprüchliches Zusammenleben von Stimmen und Bedeutungswirkungen. Dies geschieht von Anfang an, ausgehend von der Polyphonie der mütterlichen Sprache, die in einer ambivalenten Position vor Ihrem neu angekommenen Baby basiert.

Die Sprache der Mutter hat keine direkte Wirkung auf inr Baby, sondern erreicht sie durch die Wirkung, die sie in sich selbst provoziert hat (CORIAT, 2000), die die Beziehung zum Baby zu einem aufschlussreichen Feld macht, das es in eine Erfahrung des reinen Vergnügens bringen kann oder nicht. Was sich sofort auf das Baby konzentriert, ist die Stimme der Mutter, diese mütterliche Melodie, musikalische und poetische Dimension mit affektiven Werten (LAZNIK-PENOT, 2013), die das Baby einbezieht und erhält, völlig abhängig. Melodie, die die fütternde und betäubende Szene erzeugt. Das Baby legt besonderes Augenmerk auf die Stimme der Mutter und in ihrer Abwesenheit auf jede melodische Stimme, die Zuneigung hat. So komponiert er die Interaktion mit anderen in seiner Umgebung.

Diese Zuneigungsstimme ist durch Feinheiten, Dynamik und Rauheit (Variation der Sinne und Empfindungen) ein Raum für den spontanen Ausdruck des mütterlichen Unbewussten (FÓNAGY apud LAZNIK-PENOT, 2013). Aus diesem Grund ist es wichtig zu beachten, ob in dieser melodischen Rede süße Worte hervorgebracht werden, oder ob diese Stimme aus der mütterlichen Ambivalenz in ihren Geräuschen auch andere sehr seltsame Worte hervorbringen kann (Worte) sehr eigenartig (LAZNIK-PENOT , 2013, S. 130). Dies scheint ein Weg zu sein, um zu verstehen, wann die Interaktion nicht erfolgreich ist.

Die melodische Wiege der Muttersprache heißt das Baby willkommen und wirft es in die symbolische Dimension der menschlichen Existenz, wie Melgaço (2013, S.10) warnt, wenn er sagt, dass der Zivilisationsprozess die Macht der Worte hervorhebt.

Zur Stimme der Mutter präsentiert sich das Baby mit seinem Aussehen, seinen Bewegungen, seinen Klängen und dem Hin und Her zwischen den beiden

Verfügbar in: https://www.nucleodoconhecimento.com.br/psychologie/symbolischebindung 
Interaktionsspielen, die mit Worten die Beziehung baden, in der Mutter und Baby stehen.

Dies sind ursprüngliche konstitutive Zeiten, in denen es möglich ist, Sackgassen und, wenn sie schlüpfen, Symptome, Ausdrücke, die in Nahrung, Schlaf und Sprache fließen, zu haben, soweit diese Funktionen zentral die Beziehung zwischen dem Baby und seiner Mutter betreffen.

Insofern und schließlich liegt der Kern der Frage weder in der Mutter noch in ihrem Baby, sondern in der Beziehung zwischen ihnen, in einem Prozess nicht der Ähnlichkeit, sondern der Identifikation, der zunächst dadurch gekennzeichnet ist, dass, wenn Babys immer noch nicht sprechen, dieser Beziehungsumfang vor allem durch die mentalen Prozesse der Mutter zerstört wird, was das erste Mal der Konstitution der psychischen Logik wäre und wie Vorcaro (2005, S. 24) hervorhebt, entsteht aus dem signifikanten Schwarm, der im Feld des Anderen produziert wird, in den das Lebewesen eingetaucht ist, der "vorherige Ort" des Subjekts als Wirkung der Sprache. So ist das, was bei der Mutter unbewusst bleibt, anfälliger für Inschriften und Offenbarungen des Babys (CHAVES, 2013, S. 228).

Schlafen, Essen und, warum nicht darauf zeigen, Anschauen/Angeschaut werden die ersten „Worte“ des Babys - sind die Bühne für Offenbarungen für die Mutter und inr Baby. In dieser einzigartigen Beziehung hängt die Entwicklung der Säulenfunktionen im Leben des Kindes von dem langen Weg zwischen der Objektposition (von und für die Mutter) und der des Subjekts ab.

\section{ENDGÜLTIGE ÜBERLEGUNGEN}

Wie bereits erwähnt, stammt diese Studie aus der Klinik und wir schlagen vor, dass sie zu ihr zurückkehren sollte. Das heißt, angesichts von Beschwerden im Zusammenhang mit Schlaf-, Ess- und Sprachstörungen ist es relevant, dass der Arzt in der Lage sein sollte, sich um die möglichen Anforderungen zu kümmern, die sich

RC: 98237

Verfügbar in: https://www.nucleodoconhecimento.com.br/psychologie/symbolischebindung 
aus der symbolischen Artikulation zwischen diesen drei Dimensionen ergeben; Artikulation, die dem menschlichen Funktionieren innewohnt, wenn die Untrennbarkeit zwischen Sprache, Körper und Psychismus angenommen wird.

Wenn ja, kann der Traum vielleicht auch in zukünftige Studien über die komplizierte symbolische Implikation einbezogen werden, die hier gearbeitet wird.

\section{VERWEISE}

ANZIEU, D. O Eu-pele. São Paulo: Casa do Psicólogo, 1989.

BLAIR, O. S. O co-leito em perspectiva. Jornal de pediatria, 84(2), 99-101, 2008.

CABALLO, V. E., NAVARRO, J. F. e SIERRA, J. C. Tratamento comportamental dos transtornos do sono In: CARLSON, N. R. Fisiologia do Comportamento. 7ed, SP: Manole, 2002.

Cabassu G. Palavras em torno do berço. In: Wanderley D. B. (org) Palavras em torno do berço. Salvador, Ágalma, 2003.

CHAVES, M. P. C. T. O lugar do analista na clínica com bebês. In: BUSNEL M. C. e MELGAÇO R. G. (orgs) O bebê e as palavras: uma visão transdisciplinar sobre o bebê. Instituto Langage, 2013.

CISMARESCO, A. S. O grito neonatal e suas funções. (seção: o grito e as reações fisiológicas e emocionais das mães). In: BUSNEL, M. C. A linguagem dos bebês. São Paulo, Escuta ed., 1997.

CORIAT, E. Os flamantes bebês e a velha psicanálise. Estilos da Clínica. V.5, n.8, 2000.

FARIAS, C. N. F. e GOMES DE LIMA, G. Relação mãe-criança: esboço de um percurso na teoria psicanalítica. Revista Estilos da Clínica, ano IX, n. 16, 2004.

$\mathrm{RC}: 98237$

Verfügbar in: https://www.nucleodoconhecimento.com.br/psychologie/symbolischebindung 
FÉDIDA, P. Le conte et la zone de l'endormissement. In: Corps de vide et espace de séance. Paris, Jean Pierre Delaye, 1977.

FOLINO, C. S. G. e LOPES DE SOUZA, AS. As Reverberações do encontro mãebebê: sobre a depressão e a depressividade pós-parto. In: BUSNEL, M. C. E MELGAÇO, R. G. (orgs) $O$ bebê e as palavras: uma visão transdisciplinar sobre o bebê. Instituto Langage, 2013.

GEIB, L. T. C., CATALDO NETO,A, WAINBERG R, NUNES ML. Sono e envelhecimento. Rev. Psiquiatria. Rio Gd. Sul, 25(3):453-465, 2003.

GEIB, L. T. C. Desenvolvimento dos estados do sono na infância. Revista Brasileira de Enfermagem, 60(3):323-26, 2007.

GROMANN, R. M. G. Sonho e psiquismo: o labirinto entre o corpo e a subjetividade. Pulsional Revista de psicanálise, 164: 27-34, 2002

JERUSALINSKY, A. Psicanálise e desenvolvimento infantil. Porto alegre, Artes e Ofícios, 2004.

JOHN, M. W. Sensitivity and specificity of the multiple sleep latency test (MSLT), the maintenance of wakefulness test and the Epworth sleepiness scale: failure of the MSLT as a gold standard. J. Sleep Res, 9: 5-11, 2000.

LAZNIK-PENOT, M. C. Rumo à palavra. São Paulo, Escuta ed., 1997.

LAZNIK-PENOT, M. C. Linguagem e comunicação do bebê de zero a três meses. In: LAZNIK-PENOT, M. C. A hora e a vez do bebê. Instituto Langage, 2013.

LEITE, C. A. O. Quando o corpo pede um nome. Tese de doutorado, Instituto de Estudos da Linguagem, UNICAMP, 2008.

$\mathrm{RC}: 98237$

Verfügbar in: https://www.nucleodoconhecimento.com.br/psychologie/symbolischebindung 
MADEIRA, I. R. e AQUINO, L. A. Problemas de abordagem difícil: "não come" e "não dorme". J Pediatr, 79 (Supl 1): 43-54, 2003.

MELGAÇO, R. G. Prefácio. In: BUSNEL, M. C. e MELGAÇO, R. G. (orgs) O bebê e as palavras. São Paulo, Instituto Langage, 2013.

PALLADINO, R. R. R.; CUNHA, M.C. e SOUZA, L. A. P. Transtornos de linguagem e transtornos de alimentação em crianças. Revista Psicanálise e Universidade, 21: 95-108, 2004.

PALLADINO, R. R. R.; CUNHA, M.C. e SOUZA, L. A. P. Transtornos de linguagem e de alimentação: coincidências ou co-ocorrências? Pró-fono Revista de Atualização Científica, 19, 205-214, 2007.

PALLADINO RRR. Linguagem e sono. Anais do XXIV Congresso Brasileiro de Fonoaudiologia, Sociedade Brasileira de Fonoaudiologia, São Paulo, 2016

PALLADINO RRR. Sono e alimentação: funções psíquicas associadas. Anais do III Congresso Iberoamericano de Fonoaudiologia e XXVI Congresso Brasileiro de Fonoaudiologia. Sociedade Brasileira de Fonoaudiologia, Curitiba, 2018.

PENHA, N. C. G. Dormir nos braços da mãe: a primeira guardiã do sono. $\operatorname{Rev}$ Psichê, 6(10):65-84, 2002.

PIAULINO DE ARAÚJO, P. D. Validação do questionário do sono infantil de Reimão e Lefèvre (QRL). Tese de doutorado, Departamento de Neurologia, USP, 2012.

RÉÉDUCATION ORTHOPHONIQUE, 44 année, juin/2006, trimestriel n. 226 - La deglutition Dysfunctionnelle, 2006.

RC: 98237

Verfügbar in: https://www.nucleodoconhecimento.com.br/psychologie/symbolischebindung 
SANTOS, M. C. Problemas alimentares da infância sem diagnóstico clínico: quando vigiar, quando atuar? Rev. Hospital de Crianças Maria Pia, vol. XIII, n.4: 342-7, 2004.

SERVILHA, B. e RAAD BUSSAB, V.S. Interação Mãe-Criança e Desenvolvimento da Linguagem: a Influência da Depressão Pós-Parto. Psico, Porto Alegre, v. 46, n. 1, pp. 101-109, jan.-mar. 2015.

SPINOZA, B. Ética. Trad. e notas de Thomaz Tadeu. Belo Horizonte, Autêntica Ed. 2007.

STORK, H.; LY, O. e MOTA. G. Os bebês falam: como você os compreende? Uma comparação intercultural. In: BUSNEL, M. C. (org) A linguagem dos bebês. Sabemos escutá-los? São Paulo: Escuta Ed., 1997.

THIBAULT, C. A língua, órgão chave das oralidades. Rééducation Orthophonique, 44 année, juin/2006, trimestriel, n.226 - La deglutition Dysfunctionnelle, p.115, 2006.

VORCARO, A. Crianças em Psicanálise, Rio de Janeiro, Companhia de Freud, 2005.

WINNICOTT, D. W. Pensando sobre crianças. Porto Alegre, Artmed, 1975.

Eingereicht: September 2021.

Genehmigt: September 2021.

RC: 98237

Verfügbar in: https://www.nucleodoconhecimento.com.br/psychologie/symbolischebindung 